\title{
Intravitreal ranibizumab as primary treatment for neovascular membrane associated with idiopathic juxtafoveal retinal telangiectasia
}

\author{
Lazaros Konstantinidis • Irmela Mantel • \\ Leonidas Zografos • Aude Ambresin
}

Received: 27 April 2009/Accepted: 1 June 2009/Published online: 16 June 2009

(C) Springer-Verlag 2009

\section{Dear Editor,}

Proliferative idiopathic juxtafoveal retinal telangiectasia (PIJRT) is characterized by bilateral ectatic lesions of the perifoveal capillaries associated with subretinal neovascularization (SRNV) [1], and has a poor natural history. [2]

It has been hypothesized that vascular endothelial growth factor-A (VEGF-A) might play an important role in the pathogenesis of PIJRT. [3] Ranibizumab (Lucentis ${ }^{\circledR}$, Genentech, South San Francisco, CA, USA) neutralizes all active forms of VEGF-A, and has been found to be effective in the treatment of exudative age-related macular degeneration. However, as far as we are aware, this is the first case to report efficacy of ranibizumab as monotherapy in the treatment of PIJT.

A 56-year-old woman was admitted to our hospital with progressive visual loss over 1 week in her right eye. Bestcorrected visual acuity (BCVA) at presentation was 0.2 (decimal equivalent). Fluorescein angiography (FFA) showed dilated, ectatic capillaries involving the temporal juxtafoveolar retina, with late exudation in both eyes and evidence of active SRNV in the right eye (Fig. 1).

Optical coherence tomography (OCT) (Carl Zeiss Meditec, Inc.) of both eyes revealed increased thickness of the temporal parafoveal retina and foveal cystoid spaces. In the right eye, OCT revealed a hyper-reflective lesion at inner retinal layers on the temporal juxtafoveal retina corresponding to the SRNV (Fig. 2).

L. Konstantinidis · I. Mantel · L. Zografos · A. Ambresin $(\bowtie)$ Hôpital Ophtalmique Jules Gonin, University of Lausanne,

15 Av. de France,

CH-1004 Lausanne, Switzerland

e-mail: aude.ambresin@fa2.ch
Following informed written consent, ranibizumab $(0.5 \mathrm{mg})$ was administered intravitreally. The patient was followed monthly with OCT and FFA. Reinjection criteria were evidence of SRNV activity on FFA.

One month after treatment, BCVA improved to 0.32 (decimal equivalent), and central macular thickness (CMT) on OCT decreased. Exudation from both the SRNV and the ectatic vessels was decreased but still persistent on FFA.

Two more injections at monthly intervals were administered in order to obtain complete regression of the neovascular complex.

At 4-month follow-up and after three injections at monthly intervals, FFA revealed no activity of the SRNV. BCVA improved to 0.5 (decimal equivalent). FFA showed regression of the neovascular complex with absence of exudation and, additionally, marked regression of the ectatic dilatation of the parafoveal vessels (Fig. 1).

At 6-month follow-up, 2 months after the third injection, BCVA decreased to 0.4 (decimal equivalent). OCT demonstrated cystoid foveal cysts, and FFA showed leakage from the ectatic vessels but no signs of SRNV activity (Figs. 1 and 2). Patient was not re-treated, and BCVA, FFA and OCT findings remained stable until the last follow-up at 8 months.

PIJT has been reported to have a poor natural history, with $80 \%$ of cases ending with a final visual acuity of 0.1 decimal equivalent or worse [2].

Several treatment modalities have been assessed $[4,5]$, with poor results in most of the cases. Recently, several studies have shown favorable functional and anatomic results using the off-label anti-VEGF-A drug bevacizumab in the treatment of PIJT [3, 6, 7]. In 2008, Rishi et al. reported a case of PIJT that underwent primary treatment with a combination of photodynamic therapy (PDT) and intravitreal ranibizumab that demonstrated functional improvement [8]. 


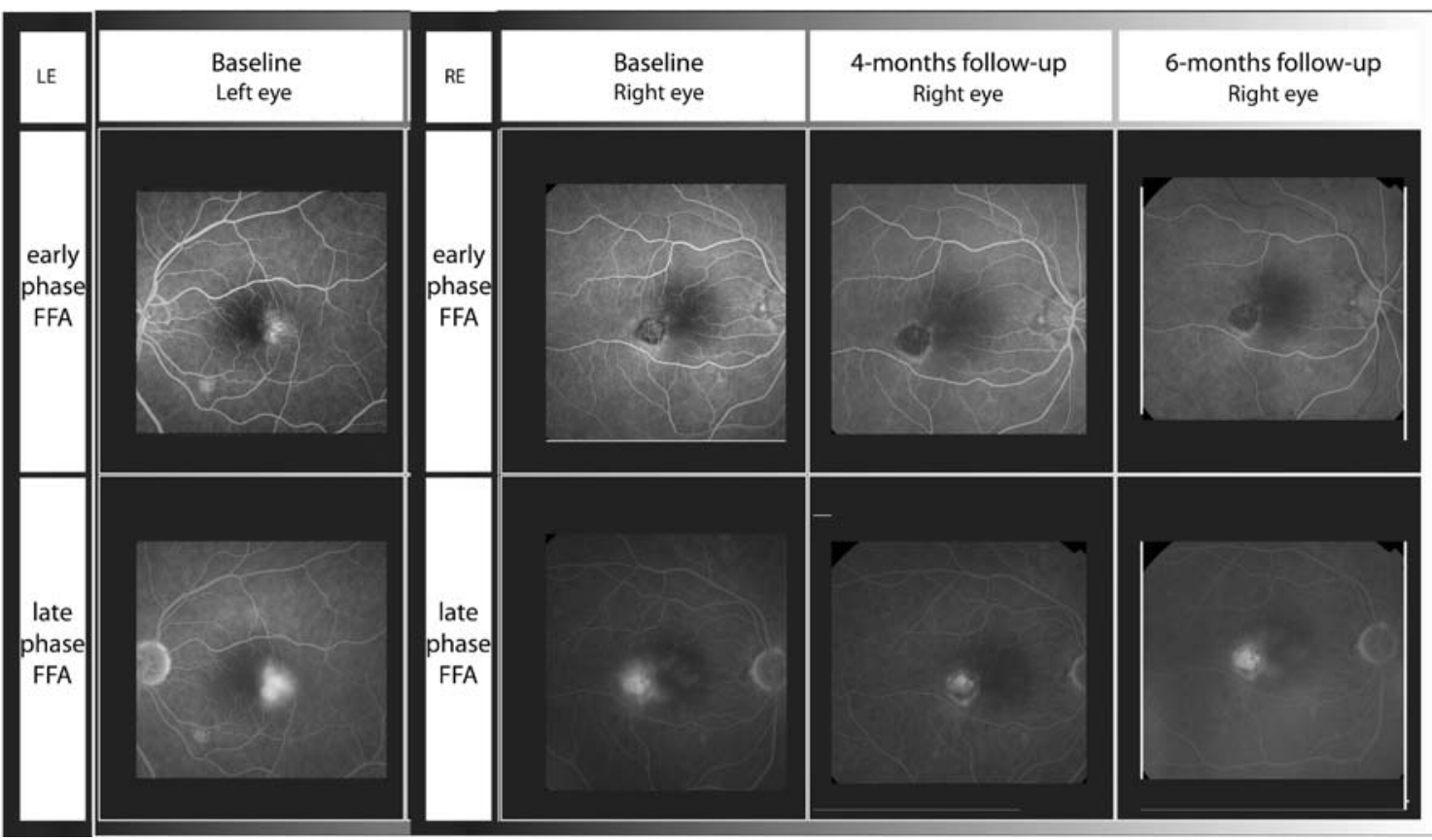

Fig. 1 Angiography findings of the left eye at baseline and the right eye at baseline, at 4-month follow-up and at 6-month follow-up. Baseline (left eye): FFA shows dilated, ectatic capillaries involving the temporal juxtafoveolar retina with late exudation. Baseline (right eye): FFA shows dilated, ectatic capillaries involving the temporal juxtafoveolar retina with late exudation and evidence of active SRNV. Four-month follow-up (right eye): FFA shows absence of exudation from SRNV and additionally, marked regression of the ectatic dilatation of the parafoveal vessels. Six-month follow-up (right eye): FFA shows leakage from the ectatic vessels but no signs of SRNV activity

\section{4-months follow-up}
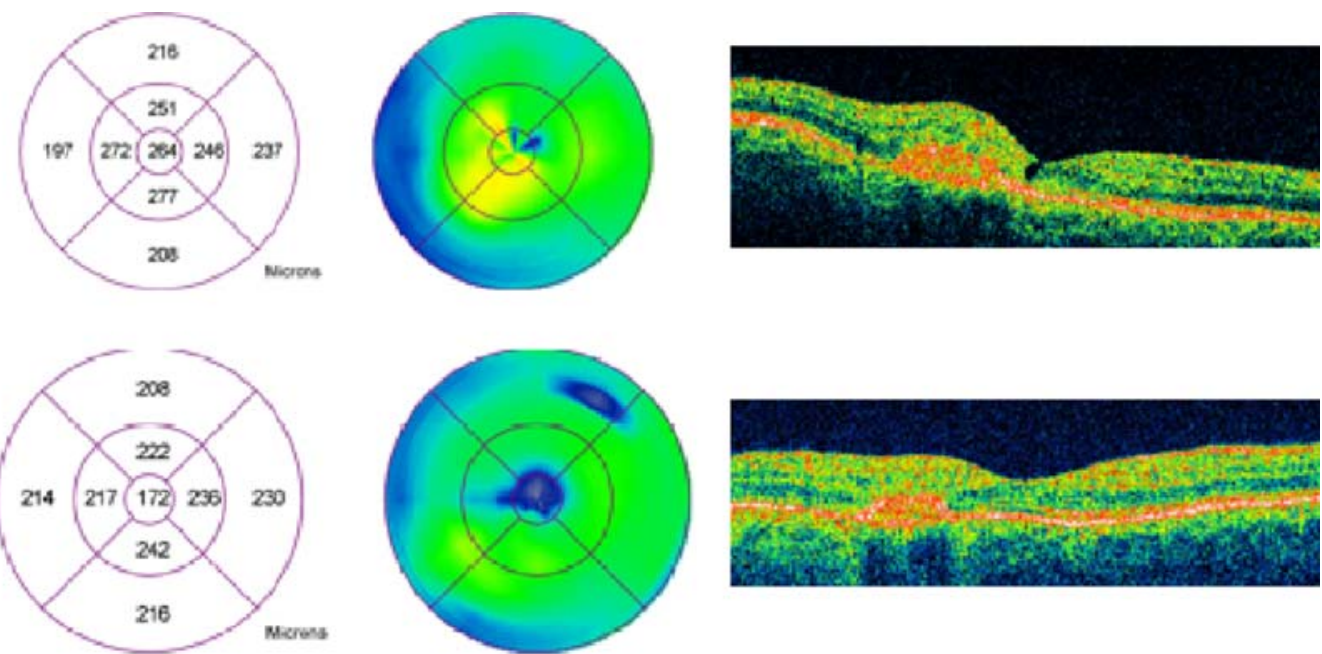

\section{6-months follow-up}
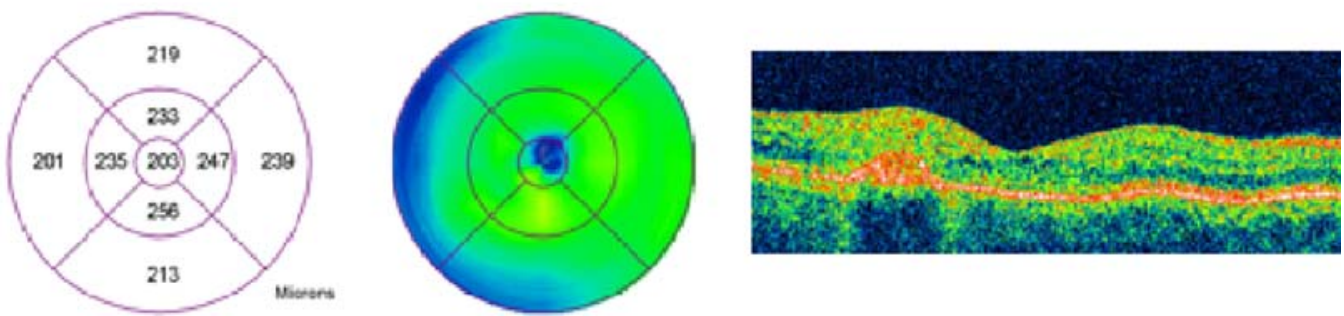

Fig. 2 OCT findings (macular map and horizontal macular scan) of the right eye at the baseline, at 4-month follow-up and at 6-month follow-up. Baseline: OCT reveals increased thickness of the temporal parafoveal retina and a foveal cystoid space. Note the hyper-reflective lesion at inner retinal layers on the temporal juxtafoveal retina corresponding to

the SRNV. Four-month follow-up: OCT shows significant decrease of CMT. Six-month follow-up: At 6-month follow-up, 2 months after the third injection, OCT demonstrated increased CMT and small cystoid foveal cysts 
In our case, treatment with three monthly interval intravitreal injections of ranibizumab resulted in an improvement of BCVA and complete resolution of SRNV during the follow-up period. However, effect of ranibizumab on ectatic vessel activity seemed to be more transient in this case. FFA appeared to be a very useful exam to evaluate treatment efficacy to SRNV activity.

Ranibizumab may be an effective treatment of PIJT; however, further studies are warranted to determine more precisely its clinical benefit and its safety in the treatment of PIJT.

Financial Support None

\section{References}

1. Yannuzzi LA, Bardal AM, Freund KB, Chen KJ, Eandi CM, Blodi B (2006) Idiopathic macular telangiectasia. Arch Ophthalmol 124:450460
2. Engelbrecht NE, Aaberg TM Jr, Sung J, Lewis ML (2002) Neovascular membranes associated with idiopathic juxtafoveolar telangiectasis. Arch Ophthalmol 120:320-324

3. Kovach JL, Rosenfeld PJ (2009) Bevacizumab (avastin) therapy for idiopathic macular telangiectasia type II. Retina 29:27-32

4. Snyers B, Verougstraete C, Postelmans L, Leys A, Hykin P (2004) Photodynamic therapy of subfoveal neovascular membrane in type 2a idiopathic juxtafoveolar retinal telangiectasis. Am J Ophthalmol 137:812-819

5. Berger AS, McCuen BW 2nd, Brown GC, Brownlow RL Jr (1997) Surgical removal of subfoveal neovascularization in idiopathic juxtafoveolar retinal telangiectasis. Retina 17:94-98

6. Jorge R, Costa RA, Calucci D, Scott IU (2007) Intravitreal bevacizumab (avastin) associated with the regression of subretinal neovascularization in idiopathic juxtafoveolar retinal telangiectasis. Graefes Arch Clin Exp Ophthalmol 245:1045-1048

7. Mandal S, Venkatesh P, Abbas Z, Vohra R, Garg S (2007) Intravitreal bevacizumab (avastin) for subretinal neovascularization secondary to type 2a idiopathic juxtafoveal telangiectasia. Graefes Arch Clin Exp Ophthalmol 245:1825-1829

8. Rishi P, Shroff D, Rishi E (2008) Combined photodynamic therapy and intravitreal ranibizumab as primary treatment for subretinal neovascular membrane (srnvm) associated with type 2 idiopathic macular telangiectasia. Graefes Arch Clin Exp Ophthalmol 246:619-621 